\title{
USE OF POLLEN MORPHOLOGY TRAITS FOR IDENTIFYING SPECIES OF CENTAUREA L. ASTERACEAE IN KURDISTAN-IRAQ
}

\author{
Rizgar Y. Ismail ${ }^{\mathrm{a},}$ *, Saleem E. Shahbaz \\ ${ }^{a}$ Dept. of Biology, Faculty of Science, University of Zakho, Zakho, Kurdistan Region, Iraq - (rizgar.ismail@uoz.edu.krd) \\ ${ }^{\mathrm{b}}$ Dept. of Agricultural Engineering, Faculty Science,University of Duhok, Kurdistan Region, Iraq (saleem.shahbaz@uod.ac)
}

\begin{abstract}
:
The polleniferous material of Centaurea taxa grow in Kurdistan-Iraq was collected. Samples were tested using scanning electron microscopy (SEM) and light microscopy (LM). In the polar view, pollen grain outlines are mostly triangular or triangular-circular. In equatorial view, the elliptic shape is common, while circulars or semicircular shapes are rare. The exine sculpture is scabrate, and the tectum is perforate. Number of sculpture microspinules/ $\mu \mathrm{m}^{2}$, and their heights together with the number of wall perforations provide significant characters for taxa delimitation. Pollen grains of all species are of medium size, very few of some species pollen are larger or smaller in size. Species producing subprolate pollen shape is common, while oblate spheroidal and Prolate spheroidal shapes are less common. The principal component analysis and the discriminant analysis can recognize $65.478 \%$ and $87.54 \%$ of the total variability respectively; moreover, the discriminant function can correctly classify 125 or $78.125 \%$ out of 160 observations for pollen traits.
\end{abstract}

KEYWORDS: Centaurea L., Microspinules, Suboblate, Prolate, Centaurea regia.

\section{INTRODUCTION}

Centaurea $L$. is one of the largest genera of Asteraceae with 250-700 species of herbaceous annual, biennial and perennial plants (Bancheva et al., 2014; Erkara et al., 2012; Shabestari et al., 2013; Susanna \& Garcia-Jacas, 2007; Tasar et al., 2018). Species of the genus are found in the northern Hemisphere, mostly in the Eastern part of the Hemisphere; the Middle east and nearby areas is species-rich. Distribution is predominantly of old world ranges (Bancheva et al., 2014; Bremer, 1994; Dittrich, 1977).

The laboratories dealing with palynological traits increased the diversity of pollen grains and using their results in plant taxonomy. Such laboratories increased dramatically our knowledge on morphological diversity of pollen grains and spores than previously, but their influence on plant taxonomy is still less than it should have done. Some of the reasons why the taxonomic impact of palynology has been less than it could have been are identified. Firstly, studies of pollen have not always been integrated with the results obtained from other kinds of studies (Blackmore \& Barnes, 1991). Secondly, modern taxonomic methods of character analysis have often not been used. Therefore, palynology has tended to be left behind while systematics has moved on. According to Blackmore (1996), there are two more factors that tend to reduce the impact of palynology on systematic: the complex terminology of the subject (Davis \& Heywood, 1963) and the extreme complexity of circumscribing comparative characters based on pollen grains morphology.

The knowledge of pollen morphology can be used as an instrument of multiple scientific researches in systematic botany, paleobotany, paleoecology, pollen analysis, aeropalynology, criminology, allergy, stratigraphic correlation of oil-bearing rocks and coal fields, drugs in the field of medicopalynology and improvement of honey in the field of mellittopalynology and copropalynology (Meo \& Khan, 2003).
Palynology is one of the most widely used research tools in quaternary studies (Edwards, 1983). It plays an important role in plant systematic studies. Moreover, as indicated by (Edwards \& Macdonald, 1991; Prentice, 1988) data derived from pollen studies can be used to provide an indication as to the response of natural vegetation to human impacts through history, and to climatic and environmental changes. Huntley (1990) recorded data for pollen strengthen predictions of how vegetation is likely to respond to future climatic conditions, thereby providing an indication of the future agricultural and silvicultural potential of various regions.

Pollen morphology provides significant evidences to support the separation of the taxa at different levels of hierarchy. For example pollen provides the primary basis for recognition of subfamilies in the Labiatae, but at the tribal level only the Ajugeae has distinctive pollen. Several genera, notably Collinsonia, Salvia, Teucrium, and Trichostema, have pollen that is very different from other genera. At the infrageneric level, pollen provides valuable taxonomic characters in several genera, notably Hyptis, Monardella, Salvia, Stachys, Teucrium, and Trichostema (Binzat et al., 2014).

Pollen morphology of 19 taxa of the Centaurea in Iran was investigated by light and scanning electron microscope (SEM). Examination showed pollen grains to be tricolporate, isopolar, radially symmetrical, oblate spheroidal, prolate spheroidal, subprolate, tectum perforate and scabrate. Based on the exine ornamentation, two types of pollen grains were recognized: 1 . dense acute spinules and 2. sparse spinules (Shabestari et al., 2013).

Some endemic Turkish species (Centaurea antalyense $\mathrm{H}$. Duman \& A. Duran, C hierapolitana Boiss., Heimerl, C. lycia Boiss., C. tossiensis Freyn and C. wagenitzii Hub.-Mor) were studied under light and electron microscopy (SEM), investigations showed that the pollen grains are more or less

\footnotetext{
* Corresponding author

This is an open access under a CC BY-NC-SA 4.0 license (https://creativecommons.org/licenses/by-nc-sa/4.0/)
} 
spheroidal-subprolate, tricolporate and the exine sculpture is tecate-scabrate (Erkara et al., 2012).

The aim of this study in Kurdistan-Iraq is to provide additional knowledge about the pollen morphology of the taxa under investigation and to determine the extent to which the data can be used as taxonomic character in discriminating between taxa.

\section{MATERIALS AND METHODS}

Flowering buds in the stage just before anthesis were obtained from living plants in the field, of all Centaurea species growing widely in Kurdistan region. Plant samples were dried in the laboratory conditions, and kept at $4 \mathrm{C}^{\mathrm{o}}$ for subsequent use. Preparation of the slides of pollen grains was mounted in methyl green glycerine jelly; large chunks of pollen grains may be separated if necessary by stirring ( $1 \mathrm{~g}$ methyl green dissolved in $100 \mathrm{ml}$ alcohol 95\%) (Radford et al., 1974).

The slides were observed by light microscope and photographed using digital Sony camera (18.2 mega-pixels). Morphological observations, including polar length (P) and equatorial diameter (E), $\mathrm{P} / \mathrm{E}$ ratio, number of apertures, mesocolpium in the equatorial view, apocolpium, colpus length, exine thickness, apertural shape, mean, range and standard deviation for each character was based on 30 observations for each species, Size classes of (Erdtman, 1945) were assessed, while shape classes of (Erdtman, 1952) were followed. The pollen terminology was followed by Punt et al., (2007) and Erdtman, (1971). The samples were examined and photographed by using scanning electron microscope model TESCAN MIRA 3.

\section{RESULTS AND DISCUSSION}

The pollen grains are isopolar, radially symmetrical, tricolporate, monad, Characters of the studied pollen grains of 16 Centaurea species using LM and SEM are listed in Table (3) and shown in figures (3-10).

In the polar view, pollen grain outlines are mostly triangular or triangular-circular and the colpi are acute at both ends. In equatorial view, the elliptic shape is abundant, no triangular are present, while circulars or semicircular are fewer. Tetrangular pollen shapes are only observed in polar view of both $C$. persica and $C$. tomentella. In all species examined, the ectoaperture is bigger than the endoaperture. The exine is consisting of two layers, well visible in LM.

Under LM and SEM, the exine sculpture is scabrate, psilate towards the colpi and the tectum is perforate, respectively (Figures 3-10). Pollen grains sculpture is spinulose (echinute) with spines or microspinules. Scanning electron micrograph of a pollen grain of $C$. aggregata, $C$. behen, $C$. cardunculus, and C. tomentella showing colpi with lalongate ora (Figures 3, 4, $5,10)$, although a common structure, but is useful for identification of number of Iraqi Centaurea species.

According to number of spines or microspinules the following groups can be formed Table 5:

1. Low density microspinules (spinules), $\leq 1 / \mu \mathrm{m}^{2}$ : $C$. solstitialis, C. cyanus, C. gudrunensis, C. balsamita, .C. bruguierana.

2. Medium density microspinules, $>1 \leq 2 / \mu \mathrm{m}^{2}$ : C. hyalolepis, C. cardunculus, C. behen, C. rigida, C. persica, C. regia.

3 . High density microspinules $>2 / \mu \mathrm{m}^{2}$ : C. iberica, C. virgata, $C$. aggregata, $C$. gigantea, $C$. tomentella.

Based on the number of perforation in the pollen grain tectum, the following groups may be formed:

1. Low number of wall perforations, $<4 / \mu \mathrm{m}^{2}$ : C. iberica, $C$. solstitialis, C. hyalolepis, C. cardunculus, C. rigida, $C$. tomentella, C. persica.

2. Medium number of wall perforations, $\geq 4$ to $<4.5 / \mu \mathrm{m}^{2}$ : C. regia, C. gudrunensis, C. balsamita, C. bruguierana.
3. High number of wall perforations, $\geq 4.5 / \mu \mathrm{m}^{2}:$. cyanus, $C$. behen, C. gigantea, C. virgata, C. aggregata.

Based on the height of microspinules (spinules), three types are observed:

1. Very short microspinules $<0.300 \mu \mathrm{m}: C$. cyanus, $C$. persica. 2. Medium long microspinules, $\geq 0.300 \mu \mathrm{m}$ to $<0.500 \mu \mathrm{m}: C$. iberica, C. hyalolepis, C. regia, C. cardunculus, C. behen, $C$. gudrunensis, C. rigida, C. gigantean, C. tomentella, $C$. bruguierana. C. virgata, C. balsamita, C. balsamita.

3 . Very long microspinules, $<0.500 \mu \mathrm{m}$ : C. aggregata

Using Anova (Table 3 ) for testing pollen grain traits proved to be highly significant under $1 \%$ level of probability.

Dimensions of the polar $(\mathrm{P})$ axis ranges from $22.183 \mu \mathrm{m}(C$. aggregata) to $58.483 \mu \mathrm{m}$ long (C. gigantea), with the overall average of $35.949 \mu \mathrm{m}$, while dimensions of the aquatorial (E) axis is found to range from $19.090 \mu \mathrm{m}$ (C. bruguierana) to $59.964 \mu \mathrm{m}(C$. persica $)$ with the average of $32.535 \mu \mathrm{m}$. The polar length/the equatorial diameter ranges between $0.473(C$. persica) to 1.733 (C. behen). The average is 1.141 . In case of the apoculpium, the minimum value is $26.989 \mu \mathrm{m}(C$. virgata $)$ to 57.228 (C. cardunculus), with average of $39.400 \mu \mathrm{m}$. The mesoculpium varies between $6.619 \mu \mathrm{m}(C$. gudrunensis $)$ and $27.480 \mu \mathrm{m}(C$. persica $)$ with the average of $15.357 \mu \mathrm{m}$. The colpus length is found to be $15.457 \mu \mathrm{m}$ (C. rigida) to $35.611 \mu \mathrm{m}$ (C. persica) and the average of $24.197 \mu \mathrm{m}$. the minimum exine thickness is $2.769 \mu \mathrm{m}(C$. aggregata), while the maximum is $4.697 \mu \mathrm{m}($ C. balsamita $)$ with the average of $3.377 \mu \mathrm{m}$.

According to (Erdtman, 1945), about $98 \%$ of all investigated species, are medium sized, very little number of the total pollen grains $(1.25 \%)$ of species $C$. cyanus, $C$. cardunculus, $C$. behen, and $C$. gigantea are found to contain some large-sized pollen, and only $0.312 \%$ of $C$. virgata pollen grains for (Erdtman, 1945) are considered small.

According to pollen shapes of (Erdtman, 1971) all Centaurea species of Kurdistan region could be divided into the following groups:

1. Subprolate:
C. iberica
$\mathrm{P} / \mathrm{E}=1.290$
C. cardunculus
$\mathrm{P} / \mathrm{E}=1.301$
C. behen
C. rigida
$\mathrm{P} / \mathrm{E}=1.299$
C. tomentella
$\mathrm{P} / \mathrm{E}=1.175$
C. bruguierana
$\mathrm{P} / \mathrm{E}=1.146$
C. aggregata
$\mathrm{P} / \mathrm{E}=1.209$
$\mathrm{P} / \mathrm{E}=1.226$

2. Oblate spheroidal:
C. solstitialis
$\mathrm{P} / \mathrm{E}=0.953$
C. hyalolepis
$\mathrm{P} / \mathrm{E}=1.005$
C. cyanus
$\mathrm{P} / \mathrm{E}=0.901$
C. regia
$\mathrm{P} / \mathrm{E}=0.970$
C. gudrunensis
$\mathrm{P} / \mathrm{E}=1.143$
C. balsamita
$\mathrm{P} / \mathrm{E}=1.086$
C. virgata
$\mathrm{P} / \mathrm{E}=1.053$

3. Prolate spheroidal:

4. Suboblate:

$$
\text { C. persica } \quad \mathrm{P} / \mathrm{E}=0.878
$$

5. prolate:

C. gigantea

$\mathrm{P} / \mathrm{E}=1.423$

Morphological characters of our Centaurea species, regarding grain shape and exine pores and sculpture are in concomitant with results obtained by (Shabestari et al., 2013) on the pollen grain of species grows in Iran and some of those local endemic species investigated by (Erkara et al., 2012) in Turkey.

Use of multivariate analysis for distinguishing studied Centaurea species

Principal component analysis

Two components accounting for $65.477 \%$ of the variability in the Original data are extracted. The projection of the 7 studied pollen grain characters (Table 1) onto the plane defined by the 1-2 component planes show that the first principal component 
is influenced strongly by equatorial diameter and apocolpium length, while the second principle component is highly influenced by $\mathrm{P} / \mathrm{E}$ ratio. Therefore the mentioned pollen characters are expected to determine the species.

Table 1. Principal Components Analysis, components extracted rising 160 complete cases for 7 variables

\begin{tabular}{|l|l|l|l|}
\hline $\begin{array}{l}\text { Component } \\
\text { number }\end{array}$ & $\begin{array}{l}\text { Eigen } \\
\text { value }\end{array}$ & $\begin{array}{l}\text { Percent of } \\
\text { variance }\end{array}$ & $\begin{array}{l}\text { Cumulative } \\
\text { Percentage }\end{array}$ \\
\hline 1 & 3.04282 & 43.469 & 43.469 \\
\hline 2 & 1.54061 & 22.009 & 65.478 \\
\hline 3 & 0.949065 & 13.558 & 79.036 \\
\hline 4 & 0.721919 & 10.313 & 89.349 \\
\hline 5 & 0.503371 & 7.191 & 96.540 \\
\hline 6 & 0.209464 & 2.992 & 99.532 \\
\hline 7 & 0.032748 & 0.468 & 100.000 \\
\hline
\end{tabular}

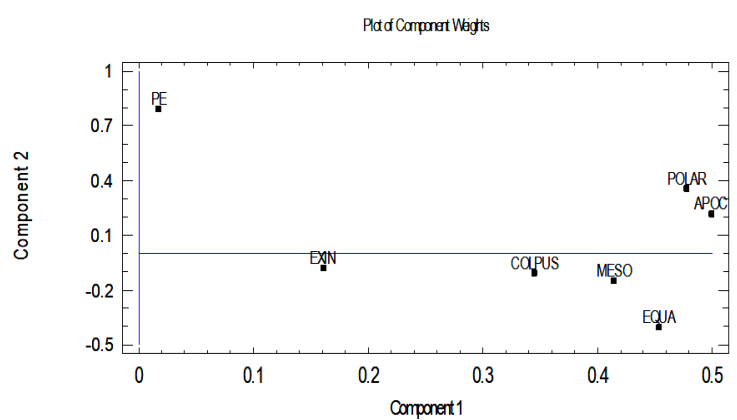

Figure 1. Principal component analyses for 7 pollen grain characters.

The first two discriminant functions (in the table 2) below account for $87.54 \%$ of the total variability, when pollen grains classified using discriminant functions, it appears that large number of pollen is assigned correctly to their original taxa. Results indicate that amongst the 160 observations used to fit the model, 125 or $78.125 \%$ were correctly classified. The projection of pollen characters onto the plane defined by the 12 discriminant functions indicates clear distinction of species (Figure 2).

Table 2. Discriminant analysis, components extracted rising 160 complete cases for 7 variables

\begin{tabular}{|l|l|l|l|}
\hline $\begin{array}{l}\text { Discriminate } \\
\text { function }\end{array}$ & $\begin{array}{l}\text { Eigen } \\
\text { value }\end{array}$ & $\begin{array}{l}\text { Relative } \\
\text { percentage }\end{array}$ & $\begin{array}{l}\text { Canonical } \\
\text { correlation }\end{array}$ \\
\hline 1 & 16.0343 & 76.12 & 0.9702 \\
\hline 2 & 2.40589 & 11.42 & 0.84047 \\
\hline 3 & 1.32664 & 6.3 & 0.75511 \\
\hline 4 & 0.633229 & 3.01 & 0.62267 \\
\hline 5 & 0.324592 & 1.54 & 0.49503 \\
\hline 6 & 0.313029 & 1.49 & 0.48826 \\
\hline 7 & 0.027660 & 0.13 & 0.16406 \\
\hline
\end{tabular}

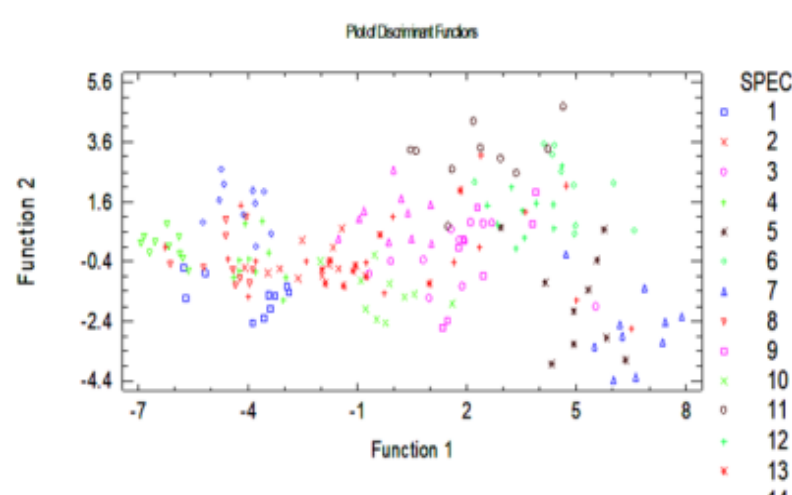

Figure 2. Discriminant analysis for identifying species.

Keys: 1. Centaurea aggregata, 2. Centaurea balsamita, 3. Centaurea behen, 4. Centaurea bruguierana, 5. Centaurea cardunculus, 6. Centaurea. cyanus, 7. Centaurea gigantea, 8 . Centaurea gudrunensis, 9. Centaurea. hyalolepis, 10. Centaurea. iberica, 11. Centaurea. persica, 12. Centaurea regia, 13. Centaurea rigida, 14. Centaurea solstitialis, 15. Centaurea tomentella, 16. Centaurea virgata.

From the overall data, it is concluded:

1. Pollen grains of all species are of medium size but very small ratio of certain species (C. cyanus, C. cardunculus, C. behen, $C$. gigantea ) contain large pollens, while only pollens of $C$. virgata contain very little number of small pollens.

2. Pollen grain outline shape in polar view of all species is mostly triangular, subtriangular, very rarely circular or semicircular. In the equatorial view, pollens are mostly more or less elliptic, seldom circular or semicircular.

3. High ratio of tetra-aperturate pollen grains in the polar view is present among pollen grains of both $C$. persica and $C$. tomentella.

4. Among pollen grain characters the shape is very useful for separating taxa. Species possessing Subprolate shape is more common, Oblate spheroidal and Prolate spheroidal shapes are coming next. The Suboblate shape $(\mathrm{P} / \mathrm{E}=0.86)$ in $\mathrm{C}$. persica and prolate shape $(\mathrm{P} / \mathrm{E}=1.423)$ in $\mathrm{C}$. gigantea are very exceptional shapes among pollen grains of Centaurea species of Kurdistan-region.

5. Principal component analysis and discriminant analysis can recognize $65.478 \%$ and $87.54 \%$ of the total variability respectively, moreover, amongst the 160 observations used to fit the discriminant function, 125 or $78.125 \%$ are correctly classified, thus can help prediction of taxa.

6 . In the pollen sculpture Microspinules height $(\mu \mathrm{m})$ and number/ $\mu \mathrm{m}^{2}$ characterized to have numbers of perforations/ $\mu \mathrm{m}^{2}$ and this character helpful to diagnostic characters for distinguishing our local Centaurea species. 
Table (3). Pollen grain characters $(\mu \mathrm{m})$

\begin{tabular}{|c|c|c|c|c|c|c|c|c|}
\hline Species & & $\begin{array}{l}\text { Polar } \\
\text { view }\end{array}$ & $\begin{array}{l}\text { Equatorial } \\
\text { view }\end{array}$ & $\begin{array}{c}\text { Polar view/ } \\
\text { Equatorial } \\
\text { view }\end{array}$ & Apoculpium & $\begin{array}{l}\text { Mesoculpiu } \\
\mathrm{m}\end{array}$ & $\begin{array}{l}\text { Colpus } \\
\text { length }\end{array}$ & $\begin{array}{c}\text { Exine } \\
\text { Thickness }\end{array}$ \\
\hline \multirow{3}{*}{$\begin{array}{c}\text { Centaurea } \\
\text { iberica }\end{array}$} & Mean & 36.365 & 28.745 & 1.290 & 40.684 & 13.831 & 22.751 & 3.159 \\
\hline & Min & 31.560 & 26.092 & 1.107 & 35.485 & 8.886 & 20.505 & 2.853 \\
\hline & $\operatorname{Max}$ & 39.915 & 31.839 & 1.441 & 44.183 & 19.148 & 24.753 & 3.834 \\
\hline \multirow{3}{*}{ C. solstitialis } & Mean & 28.619 & 30.169 & 0.953 & 32.236 & 14.535 & 21.790 & 3.282 \\
\hline & Min & 23.542 & 23.669 & 0.716 & 28.263 & 11.156 & 17.458 & 2.953 \\
\hline & $\operatorname{Max}$ & 32.559 & 34.595 & 1.248 & 36.260 & 19.387 & 25.979 & 3.665 \\
\hline \multirow{3}{*}{ C. hyalolepis } & Mean & 39.489 & 39.821 & 1.005 & 44.076 & 14.002 & 22.459 & 3.200 \\
\hline & Min & 29.469 & 27.900 & 0.756 & 39.572 & 10.203 & 19.374 & 2.905 \\
\hline & $\operatorname{Max}$ & 46.570 & 44.547 & 1.404 & 47.815 & 18.416 & 24.984 & 3.552 \\
\hline \multirow{3}{*}{ C. cyanus } & Mean & 42.786 & 43.978 & 0.901 & 45.789 & 18.532 & 29.315 & 3.417 \\
\hline & Min & 30.407 & 38.890 & 0.685 & 36.864 & 15.546 & 26.134 & 3.066 \\
\hline & Max & 51.525 & 48.409 & 1.062 & 54.509 & 20.226 & 34.370 & 3.966 \\
\hline \multirow{3}{*}{ C. regia } & Mean & 38.953 & 40.006 & 0.970 & 46.677 & 20.040 & 25.941 & 3.556 \\
\hline & Min & 35.442 & 37.332 & 0.810 & 41.915 & 14.686 & 23.526 & 3.148 \\
\hline & $\operatorname{Max}$ & 42.741 & 43.732 & 1.080 & 54.158 & 24.466 & 30.219 & 4.355 \\
\hline \multirow{3}{*}{ C. cardunculus } & Mean & 44.006 & 34.059 & 1.301 & 51.767 & 18.822 & 24.410 & 3.703 \\
\hline & Min & 37.158 & 26.329 & 1.076 & 39.658 & 12.990 & 19.318 & 3.279 \\
\hline & Max & 50.758 & 38.740 & 1.616 & 57.228 & 24.227 & 30.893 & 4.567 \\
\hline \multirow{3}{*}{ C. behen } & Mean & 39.903 & 30.663 & 1.299 & 41.979 & 12.527 & 29.864 & 3.141 \\
\hline & Min & 33.771 & 26.626 & 1.012 & 33.467 & 8.448 & 25.198 & 2.853 \\
\hline & Max & 51.279 & 36.704 & 1.733 & 48.356 & 18.729 & 34.686 & 3.365 \\
\hline \multirow{3}{*}{ C. gudrunensis } & Mean & 29.499 & 25.942 & 1.143 & 32.738 & 10.341 & 22.571 & 3.410 \\
\hline & Min & 25.160 & 21.491 & 0.891 & 30.523 & 6.619 & 20.645 & 3.066 \\
\hline & Max & 33.532 & 30.542 & 1.443 & 36.143 & 13.455 & 27.707 & 3.822 \\
\hline \multirow{3}{*}{ C. balsamita } & Mean & 32.097 & 30.200 & 1.086 & 35.900 & 11.237 & 22.103 & 3.697 \\
\hline & Min & 29.369 & 25.244 & 0.924 & 28.804 & 8.995 & 19.518 & 3.066 \\
\hline & Max & 35.394 & 35.196 & 1.302 & 37.698 & 13.270 & 24.785 & 4.697 \\
\hline \multirow{3}{*}{ C. rigida } & Mean & 35.113 & 30.103 & 1.175 & 32.097 & 16.079 & 21.436 & 3.277 \\
\hline & Min & 32.915 & 27.024 & 0.868 & 29.369 & 13.234 & 15.457 & 2.953 \\
\hline & Max & 37.345 & 42.395 & 1.349 & 35.394 & 18.402 & 24.504 & 3.907 \\
\hline \multirow{3}{*}{ C. persica } & Mean & 35.929 & 41.418 & 0.878 & 41.329 & 21.274 & 27.800 & 3.224 \\
\hline & Min & 28.357 & 25.841 & 0.473 & 34.802 & 16.116 & 24.209 & 3.066 \\
\hline & Max & 43.745 & 59.964 & 1.518 & 46.305 & 27.480 & 35.611 & 3.758 \\
\hline \multirow{3}{*}{ C. gigantea } & Mean & 49.463 & 34.748 & 1.432 & 53.447 & 20.952 & 23.67 & 3.342 \\
\hline & Min & 36.914 & 32.009 & 0.988 & 49.235 & 17.494 & 18.599 & 2.905 \\
\hline & Max & 58.483 & 38.398 & 1.678 & 56.229 & 24.408 & 29.022 & 3.719 \\
\hline \multirow{3}{*}{ C. tomentella } & Mean & 36.188 & 31.209 & 1.146 & 39.023 & 15.120 & 30.318 & 3.233 \\
\hline & Min & 32.742 & 28.503 & 1.015 & 37.026 & 6.299 & 24.611 & 2.853 \\
\hline & $\operatorname{Max}$ & 39.212 & 34.601 & 1.323 & 40.695 & 20.119 & 34.631 & 3.809 \\
\hline \multirow{3}{*}{ C. bruguierana } & Mean & 31.521 & 26.262 & 1.209 & 32.304 & 14.559 & 20.153 & 3.135 \\
\hline & Min & 27.346 & 19.090 & 0.920 & 30.157 & 12.592 & 17.207 & 2.905 \\
\hline & Max & 38.251 & 32.343 & 1.709 & 34.378 & 16.499 & 23.764 & 3.348 \\
\hline \multirow{3}{*}{ C. virgata } & Mean & 26.141 & 24.845 & 1.053 & 29.563 & 11.962 & 20.681 & 3.233 \\
\hline & Min & 24.096 & 23.312 & 0.947 & 26.989 & 9.691 & 16.952 & 2.853 \\
\hline & Max & 28.224 & 26.863 & 1.133 & 31.998 & 13.899 & 24.514 & 3.552 \\
\hline \multirow{3}{*}{ C. aggregata } & Mean & 31.104 & 25.363 & 1.226 & 36.356 & 12.541 & 19.368 & 3.109 \\
\hline & Min & 22.183 & 23.708 & 0.881 & 33.884 & 8.765 & 16.977 & 2.769 \\
\hline & Max & 38.853 & 28.464 & 1.639 & 39.193 & 15.423 & 20.720 & 3.365 \\
\hline
\end{tabular}


Table 4. Analysis of variance for pollen grain characters $(\mu \mathrm{m})$

\begin{tabular}{|c|c|c|c|c|}
\hline $\begin{array}{l}\text { Source of } \\
\text { variation }\end{array}$ & $\begin{array}{l}\text { Degree } \\
\text { of } \\
\text { freedom }\end{array}$ & $\begin{array}{l}\text { Mean } \\
\text { Square }\end{array}$ & $\begin{array}{c}\mathrm{F}- \\
\text { value }\end{array}$ & $\begin{array}{c}\mathrm{P} . \\
\text { value }\end{array}$ \\
\hline $\begin{array}{c}\text { Equatorial } \\
\text { diameter (Equa) } \\
\text { Error }\end{array}$ & $\begin{array}{c}15 \\
227\end{array}$ & $\begin{array}{l}428.463 \\
8.69934\end{array}$ & 49.25 & 0 \\
\hline $\begin{array}{l}\text { Polar length } \\
\text { (Polar) } \\
\text { Error }\end{array}$ & $\begin{array}{c}15 \\
265\end{array}$ & $\begin{array}{l}690.909 \\
9.71752\end{array}$ & 71.1 & 0 \\
\hline $\begin{array}{l}\text { Apocolpium } \\
\text { (Apoc) length } \\
\text { Error }\end{array}$ & $\begin{array}{c}15 \\
277\end{array}$ & $\begin{array}{l}946.047 \\
6.84213\end{array}$ & 138.27 & 0 \\
\hline $\begin{array}{c}\text { Mesocolpium } \\
\text { (Meso) length } \\
\text { Error }\end{array}$ & $\begin{array}{c}15 \\
214\end{array}$ & $\begin{array}{c}191.12 \\
6.78252\end{array}$ & 28.18 & 0 \\
\hline $\begin{array}{l}\text { Colpus length } \\
\text { Error }\end{array}$ & $\begin{array}{c}15 \\
145\end{array}$ & $\begin{array}{l}125.639 \\
6.44935\end{array}$ & 19.48 & 0 \\
\hline $\begin{array}{l}\text { Exine thickness } \\
\text { (Exin) } \\
\text { Error }\end{array}$ & $\begin{array}{c}15 \\
144\end{array}$ & $\begin{array}{c}0.34214 \\
0.08660 \\
17\end{array}$ & 3.95 & 0 \\
\hline $\begin{array}{l}\text { PE ratio } \\
\text { Error }\end{array}$ & $\begin{array}{c}15 \\
210\end{array}$ & $\begin{array}{l}0.3531 \\
0.0217\end{array}$ & 16.26 & 0 \\
\hline
\end{tabular}

Table 5. Average microspinule number and height and perforations number for the pollen grain sculpture

\begin{tabular}{|c|c|c|c|}
\hline Species & $\begin{array}{c}\text { Number of } \\
\text { micospinules } / \mu \\
\mathrm{m}^{2} \\
\end{array}$ & $\begin{array}{c}\text { Number of } \\
\text { perforations/ } \\
\mu \mathrm{m}^{2}\end{array}$ & $\begin{array}{c}\text { Microspin } \\
\text { ule height/ } \\
\mu \mathrm{m}\end{array}$ \\
\hline $\begin{array}{c}\text { Centaurea } \\
\text { iberica }\end{array}$ & 2.5 & 3 & 0.396 \\
\hline $\begin{array}{c}C . \\
\text { solstitialis }\end{array}$ & 0.75 & 3.5 & 0.468 \\
\hline $\begin{array}{c}C . \\
\text { hyalolepis }\end{array}$ & 1.4 & 2.5 & 0.338 \\
\hline $\begin{array}{c}\text { C. } \\
\text { cyanus }\end{array}$ & 0.3 & 7 & 0.266 \\
\hline $\begin{array}{c}C . \\
\text { regia }\end{array}$ & 1.75 & 4.3 & 0.333 \\
\hline $\begin{array}{c}C . \\
\text { cardunculus }\end{array}$ & 1.5 & 3.5 & 0.367 \\
\hline $\begin{array}{c}C . \\
\text { behen }\end{array}$ & 2 & 7 & 0.35 \\
\hline $\begin{array}{c}C . \\
\text { gudrunensis }\end{array}$ & 0.7 & 4 & 0.312 \\
\hline C. balsamita & 1 & 4 & 0.459 \\
\hline $\begin{array}{c}C . \\
\text { rigida }\end{array}$ & 1.85 & 3.7 & 0.331 \\
\hline $\begin{array}{c}C . \\
\text { persica }\end{array}$ & 1.5 & 2 & 0.270 \\
\hline $\begin{array}{c}C . \\
\text { gigantean }\end{array}$ & 3.5 & 7.5 & 0.336 \\
\hline $\begin{array}{c}C . \\
\text { tomentella } \\
\end{array}$ & 3 & 3 & 0.372 \\
\hline $\begin{array}{c}C . \\
\text { bruguierana }\end{array}$ & 0.4 & 4 & 0.392 \\
\hline $\begin{array}{c}C . \\
\text { virgata }\end{array}$ & 2.5 & 4.5 & 0.456 \\
\hline $\begin{array}{c}C . \\
\text { aggregata }\end{array}$ & 2.5 & 5 & 0.867 \\
\hline
\end{tabular}
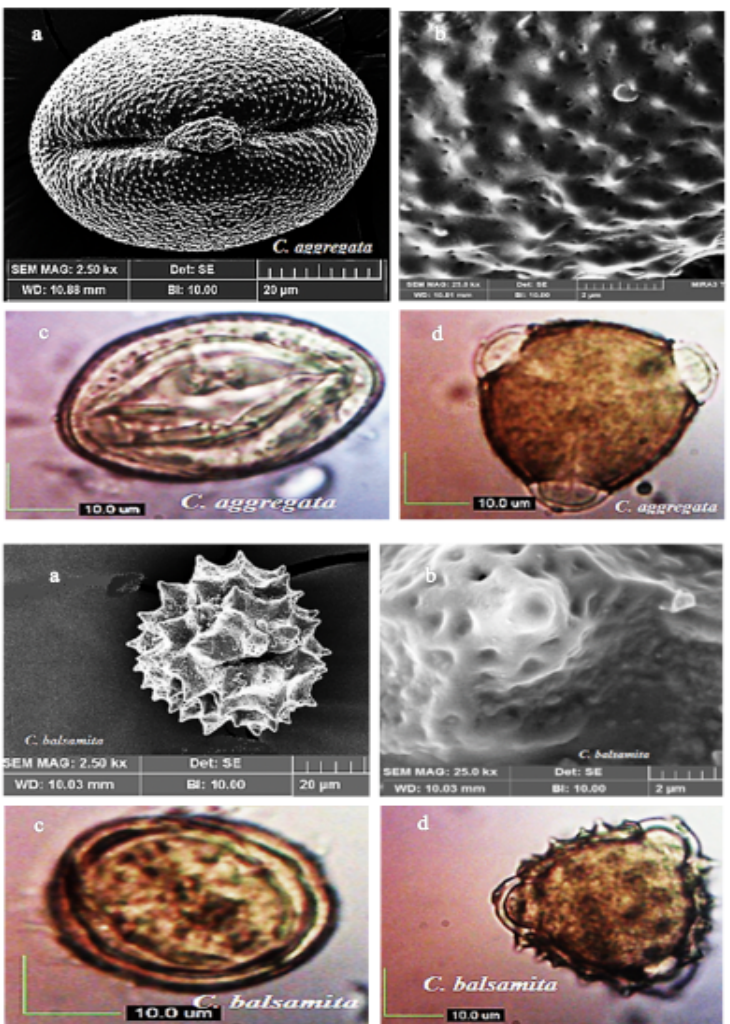

Figure 3. Centaurea aggregata, SEM: (a) equatorial view, (b) exine sculpture. LM: (c) equatorial view, (d) polar view. Centaurea balsamita, SEM: (a) equatorial view, (b) exine sculpture. LM: (c) equatorial view, (d) polar view.
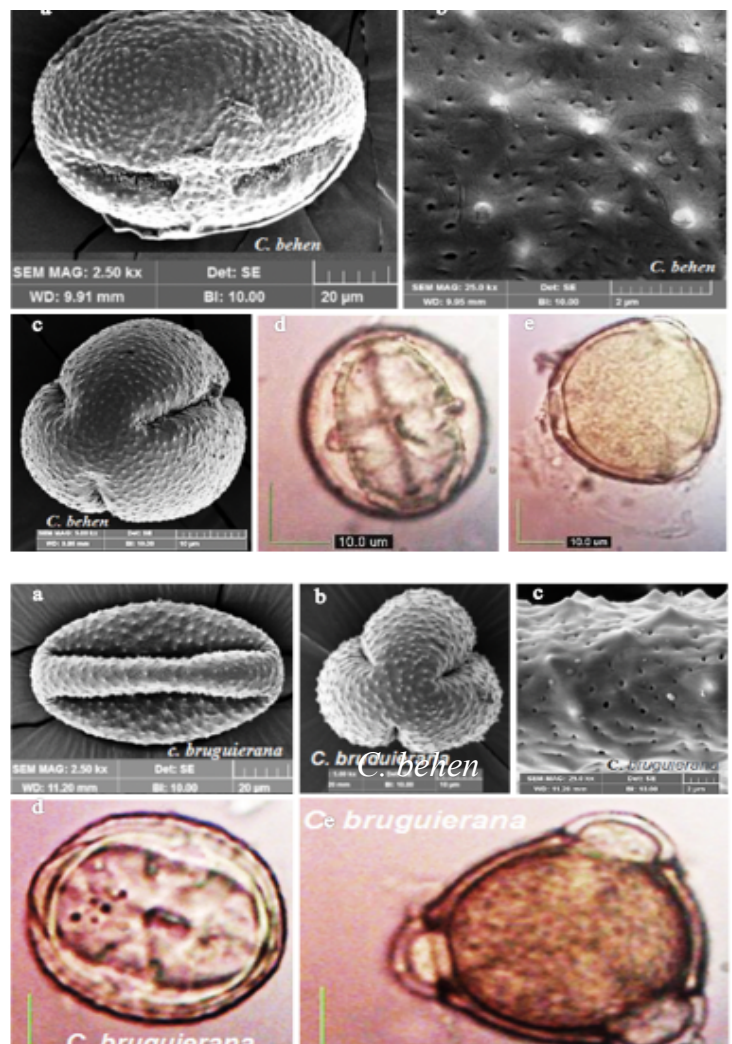

Figure (4). Centaurea behen, SEM: (a) equatorial view, (b) exine sculpture, (c) Polar view. LM: (d) equatorial view, (e) polar view. C. bruguierana, SEM: (a) equatorial view, (b) polar view (c). exine sculpture. LM: (d) equatorial view, (c) polar view. 

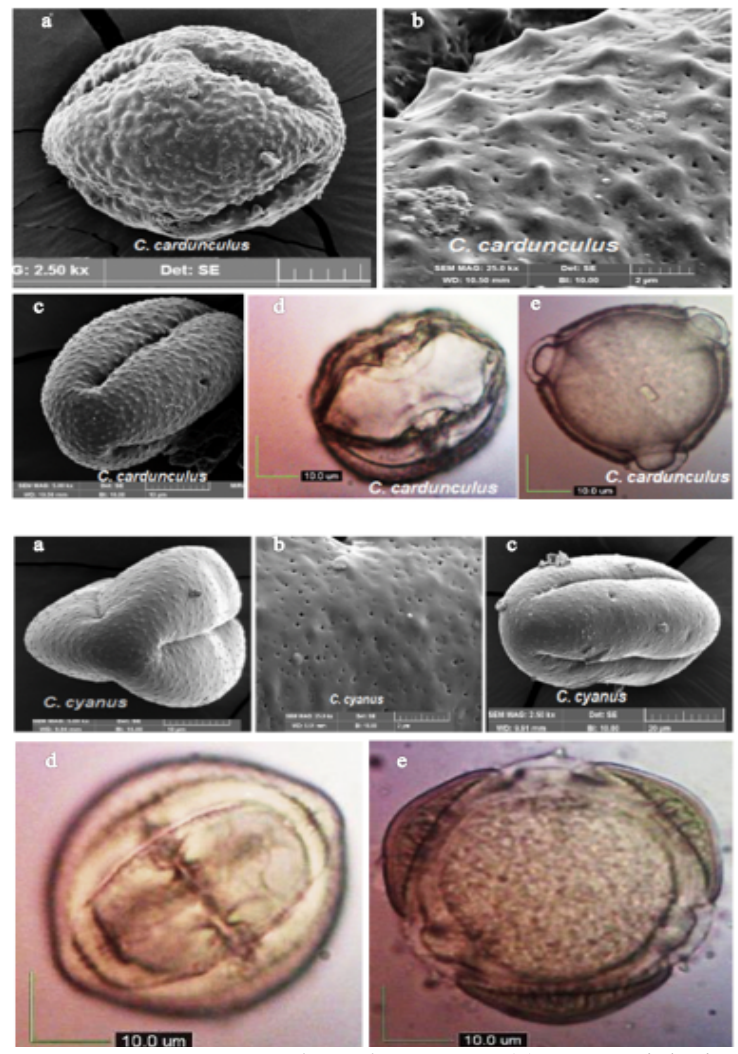

Figure 5. Centaurea cardunculus, SEM: (a) equatorial view, (b) exine sculpture, (c). Polar view. LM: (d) equatorial view, (e) polar view. C. cyanus, SEM: (a) equatorial view, (b) exine sculpture.(c) polar view LM: (d) equatorial view, (c) polar view
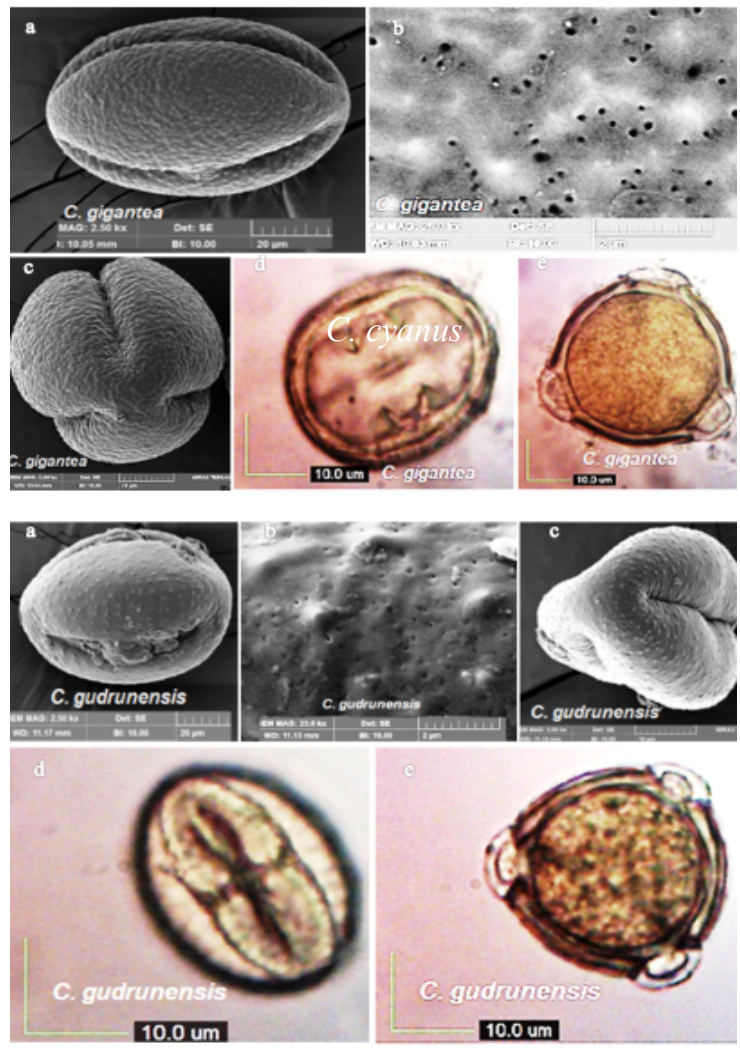

Figure 6. Centaurea gigantea, SEM: (a) equatorial view, (b) exine sculpture, (c). Polar view. LM: (d) equatorial view, (e) polar view. C. gudrunensis SEM: (a) equatorial view, (b) exine sculpture, (c) polar view LM: (d) equatorial view, (c) polar view
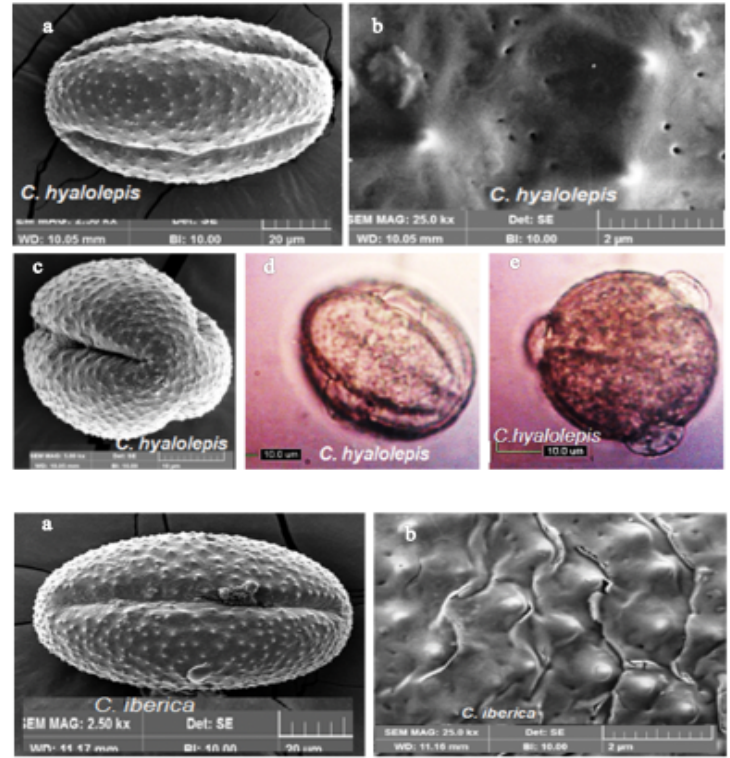

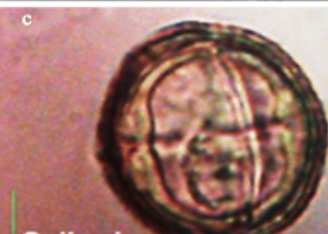

C. iberica

$10.0 \mathrm{um}$

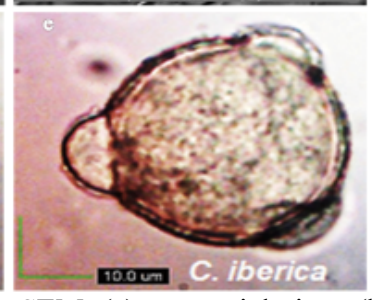

Figure 7. Centaurea hyalolepis, SEM: (a) equatorial view, (b) exine sculpture, (c). Polar view. LM: (d) equatorial view, (e) polar view. C. iberica, SEM: (a) equatorial view, (b) exine sculpture, LM: (c) equatorial view, (c) polar view.
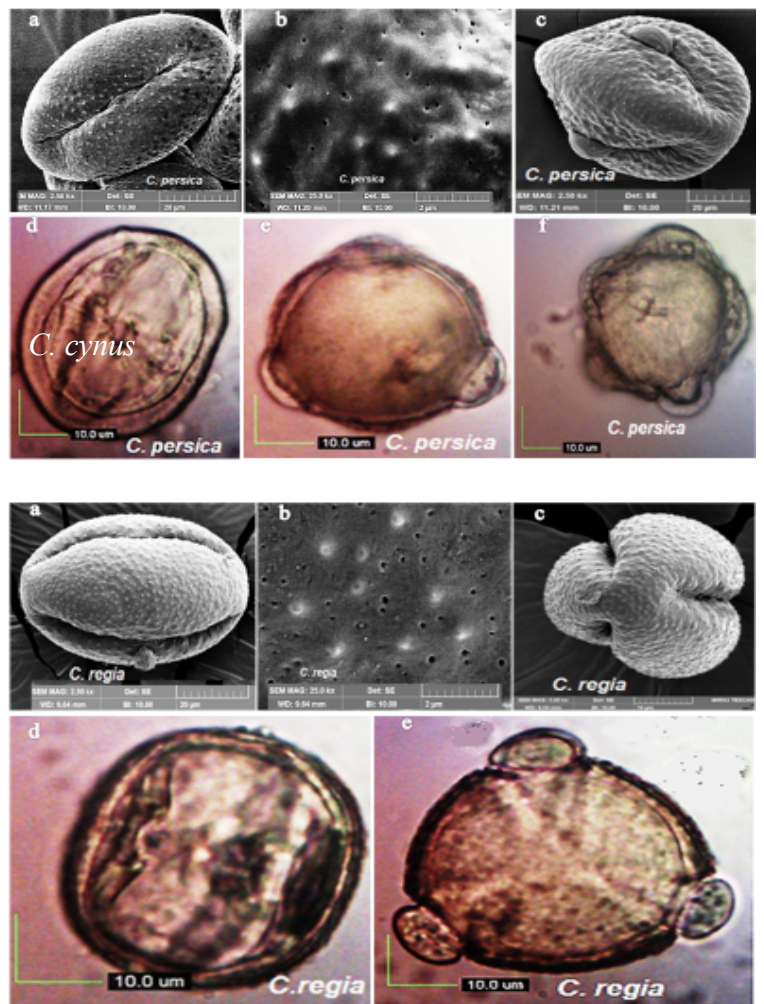

Figure 8. Centaure persica, SEM: (a) equatorial view, (b) exine sculpture, (c). Polar view. LM: (d) equatorial view, (e) polar view, (f) polar view (five apertures). C. regia, SEM: (a) equatorial view, (b) exine sculpture, (c) polar view. LM: (d) equatorial view, (e) polar view. 

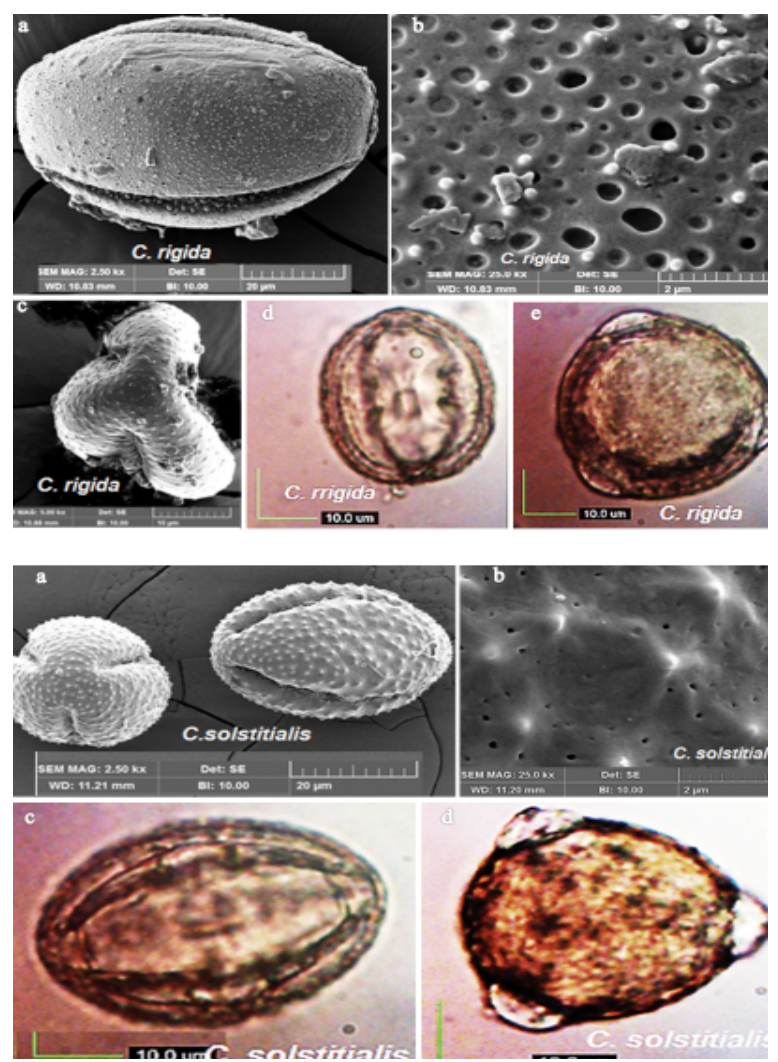

Figure 9. Centaurea rigida, SEM: (a) equatorial view, (b) exine sculpture, (c). Polar view. LM: (d) equatorial view, (e) polar view. C. solstitialis, SEM: (a) polar view, (b) equatorial view (c) exine sculpture, LM: (d) equatorial view, (e) polar view.
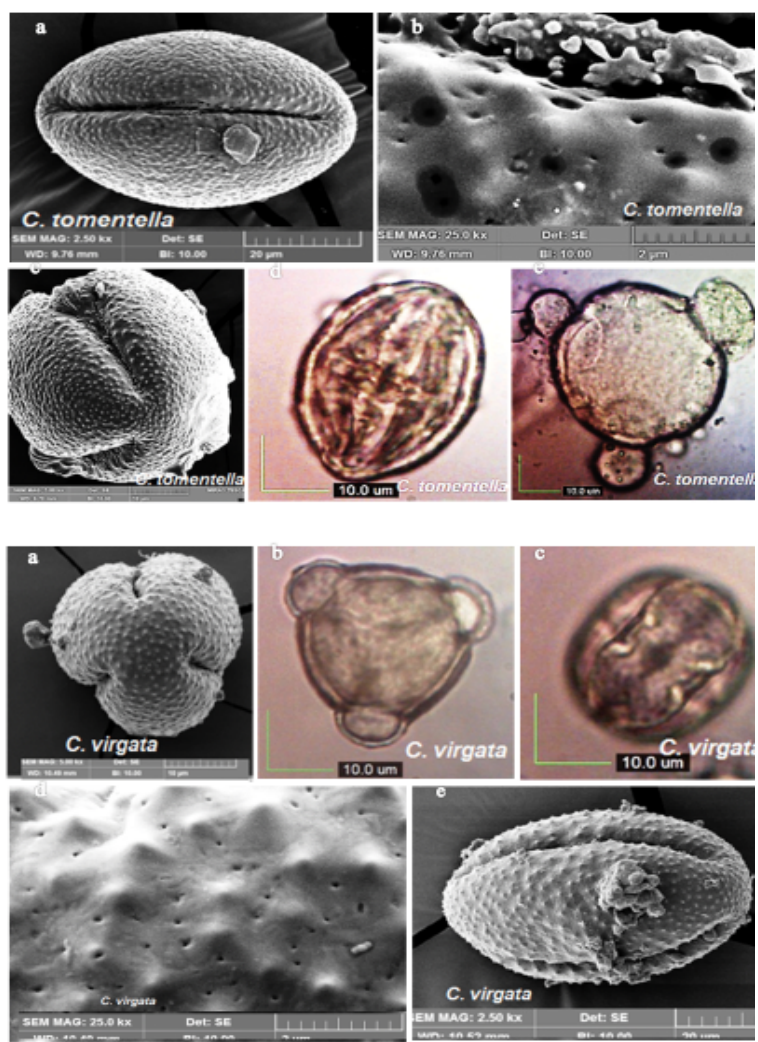

Figure (10). Centaurea tomentella, SEM: (a) equatorial view, (b) exine sculpture, (c). Polar view. LM: (d) equatorial view, (e) polar view. C. virgata, SEM: (e) equatorial view, (d) exine sculpture, (a) polar view. LM: (c) equatorial view, (b) polar view.

\section{CONCLUSION}

Among pollen grain characters the shape is very useful for separating taxa. Species possessing subprolate shape is more common, Oblate spheroidal and prolate spheroidal shapes are coming next. The Suboblate shape $(\mathrm{P} / \mathrm{E}=0.86)$ in $\mathrm{C}$. persica and prolate $(\mathrm{P} / \mathrm{E}=1.423)$ in $\mathrm{C}$. gigantea are very Exceptional shapes among pollen grains of Centaurea species. Principal component analysis and discriminant analysis can recognize $65.478 \%$ and $87.54 \%$ of the total variability respectively. In the pollen sculpture Microspinules height $(\mu \mathrm{m})$ and number $/ \mu \mathrm{m}^{2}$, moreover the number of perforations/ $\mu \mathrm{m}^{2}$ is very useful diagnostic characters for distinguishing Centaurea species.

\section{REFERENCES}

Bancheva, S., Kaya, Z., \& Binzet, R. (2014). Morphological, cytological and palynological features of three closely related Centaurea species (Asteraceae) from Turkey. Mod. Phytomorphol, 5, 79-84.

Binzat, O. K., Kahraman, A., \& Doğan, M. (2014). Pollen morphology of some taxa of Vicia L. subgenus Vicilla (Schur) Rouy (Fabaceae) from Turkey. Plant Systematics and Evolution. https://doi.org/10.1007/s00606-014-1013-0

Blackmore, S. (1996). The impact of palynology on taxonomy Bot. Macaronésica.

Blackmore, S., \& Barnes, S. H. (1991). Palynological diversity. In Pallen and Spores: Patterns of Diversification (pp. 1-8).

Bremer, K. (1994). Asteraceae, Ciadistics \& Classification. pp. Timber Press, Inc., 9999 S. W. Wilshire, Suite 124, Port land, Oregon 97225.

Davis, P. H., \& Heywood, V. H. (1963). Principles of angiosperm taxonomy. Principles of angiosperm taxonomy. Oliver \& Boyd;, 1 st edition

Dittrich, M. (1977). CynareaeĐsystematic review. In In: Heywood VH, Harborne JB, Turner BL, eds. The biology and chemistry of the Compositae. London, New York, San Francisco: Academic Press, $999 \pm 1015$

Edwards, K. J. (1983). Quaternary palynology: Consideration of a discipline. Progress in Physical Geography. https://doi.org/10.1177/030913338300700106

Edwards, K. J., \& Macdonald, G. M. (1991). Holocene palynology: II human influence and vegetation change. Progress in Physical Geography. https://doi.org/10.1177/030913339101500402

Erdtman, G. (1945). Pollen morophology and plant taxonomy. 111. Morina L., with an adition on pollen-morphology terminology. Svensk. Botany. Tidskr. 39.

Erdtman, G. (1952). Pollen morophology and plant taxonomy. Angiosperms. Chronica Botanica Co., Wathman, Massachusettes.

Erdtman, G. (1971). Pollen morophology and plant taxonomy. Hafner publishing company. New York, USA.

Erkara, I. P., Köse, Y. B., Osoydan, K., \& Yücel, E. (2012). Pollen morphology of some endemic Turkish Centaurea L. (Asteraceae, section Phaloletis) and their taxonomic value. Plant Systematics and Evolution. https://doi.org/10.1007/s00606-012-0623-7

Huntley, B. (1990). European post-glacial forests: compositional changes in response to climatic change. Journal of Vegetation Science. https://doi.org/10.2307/3235785

Meo, A. A., \& Khan, M. A. (2003). POLLEN MORPHOLOGY oF ACHILLEA (CoMPosirAE-ANTHEMoIDEAE). Pak. J. Weed Sci. Res, 9(3-4), 253-258.

Prentice, C. (1988). Records of vegetation in time and space: the principles of pollen analysis. In Vegetation history. https://doi.org/10.1007/978-94-009-3081-0_2

Punt, W., Hoen, P. P., Blackmore, S., Nilsson, S., \& Le Thomas, A. (2007). Glossary of pollen and spore terminology. Review of Palaeobotany and Palynology. https://doi.org/10.1016/j.revpalbo.2006.06.008

Radford, A. E., Dickison, W. C., Massey, J. R., \& Bell, C. R. (1974). Vascular plant systematics. Harper \& Row. New York, NY.

Shabestari, E. S. B., Attar, F., Riahi, H., \& Sheidai, M. (2013). Pollen morphology of Centaurea L. (Asteraceae) in Iran. Acta Botanica Brasilica. https://doi.org/10.1590/S0102-33062013000400004 
Susanna, A., \& Garcia-Jacas, N. (2007). Tribe Cardueae. In In J. W.Kadereit and C. Jeffrey [eds.], Flowering plant: Eudicots, Asterales,vol. VIII. In K. Kubitzki [ed.], (pp. 123-146). Springer Verlag, Berlin, Germany.

Tasar, N., Doğan, G., Kiran, Y., Oliur Rahman, M., \& Çakilcioğlu, U.

(2018). Morphological, anatomical and cytological investigations on three taxa of Centaurea L. (Asteraceae) from Turkey. Bangladesh Journal of Plant Taxonomy. https://doi.org/10.3329/bjpt.v25i2.39527. 